\title{
Accidental microembolic signals: prevalence and clinical relevance
}

Jie Chen ${ }^{1,2}$, Ying-Huan Hu' ${ }^{1}$, Shan Gao ${ }^{1}$ and Wei-Hai Xu'

\begin{abstract}
Background: The purpose of this study was to examine the occurrence of accidental microembolic signals (MES) and its clinical relevance in patients receiving routine transcranial Doppler (TCD) examinations.

Methods: We retrospectively reviewed our institutional TCD database (from January 2007-November 2012). The arteries with positive MES, the presumed sources of emboli and the clinical backgrounds were analyzed.

Results: A total of 10,067 patients received routine TCD examinations in our laboratory during the research period. MES were detected in 98 arteries of 78 patients, with a frequency of $0.77 \%$ of all the recruited patients. A high percentage of MES (64.3\%) were detected in MCAs. Sixty five (83.33\%) accidental emboli were from arterial sources, including atherosclerotic cerebral or carotid artery stenosis $(n=45)$, moyamoya disease $(n=11)$, intracranial arteries $(n=3)$ and Takayasu arteritis $(n=3)$. Thirteen (16.67 \%) emboli were from cardiac sources, including atrial fibrillation ( $n=3)$, artificial valves $(n=8)$, infective endocarditis $(n=2)$, patent foramen ovale $(n=2)$, and systemic lupus erythematosus $(n=1)$. In artificial valves disease, all patients with MES were asymptomatic, while in atherosclerotic cerebral or carotid artery stenosis, $66.67 \%(n=30)$ patients with MES were symptomatic. In different diseases with accidental MES, the proportion of symptomatic patients and asymptomatic patients were different $(p<0.001)$.
\end{abstract}

Conclusions: MES are not uncommon during routine TCD examinations, the clinical value of which varied in different diseases.

Keywords: Emboli, Microembolic signal, Stroke, Transcranial Doppler

\section{Background}

Microembolic signals (MES) - the high-intensity transient signals detected by transcranial Doppler (TCD) - have been shown to correspond to microembolic materials within the cerebral arteries [1]. MES can be detected in various clinical settings, such as carotid or intracranial stenosis, atrial fibrillation, and mechanical heart valves etc. Although most of these microemboli are clinically silent, many studies found that MES indicate increased risk of ischemic stroke, transient ischemic attack (TIA) or cognitive decline $[2,3]$.

According to the consensus on microembolus detection by TCD, the preferred recording time for patients with carotid stenosis or atrial fibrillation is at least $1 \mathrm{~h}$

\footnotetext{
* Correspondence: xuwh@pumch.cn

'Department of Neurology, Peking Union Medical College Hospital, Chinese Academy of Medical Sciences and Peking Union Medical College,

Shuaifuyuan 1, Dongcheng District, Beijing 100730, China

Full list of author information is available at the end of the article
}

[4]. However, it is difficult to monitor the arteries for such a long time for each patient. During routine TCD examinations, MES can be detected occasionally. The occurrence and its clinical relevance have remained unknown. The goal of this study was to investigate accidental MES in a large TCD database.

\section{Patients and methods}

We retrospectively reviewed our institutional TCD database for hospitalized patients from January 2007-November 2012. During this period, the presence of accidental MES in routine TCD examinations was regularly recorded. All the patients in the database were unselectively recruited, including the patients from neurology, endocrine, cardiology and surgery departments. The reasons for TCD examinations varied, depending on the clinicians' intention. According to the practice standards for TCD, the clinical indications included cerebral ischemia, intracranial arterial 
disease, detection of right-to-left shunts, sub-arachnoid hemorrhage, brain death, and periprocedural or surgical monitoring [5]. All the recruited patients underwent a careful review of previous ischemic stroke and TIA history. Thorough evaluations were performed to determine the cause of stroke or TIA, including magnetic resonance image, carotid duplex, TCD, and electrocardiogram.

\section{Ethics, consent and permissions}

The study was approved by the Peking Union Medical College Hospital Research Ethics Committee. All the patients signed a consent form.

\section{Transcranial doppler examination}

Cerebral arteries were examined with 2-MHz Doppler instrument (Pioneer TC-8080 and Companion III, NicoletEME) according to standard protocol, including middle cerebral arteries (MCA), anterior cerebral arteries (ACA), posterior cerebral arteries (PCA), and $\mathrm{C} 1$ segment of the internal carotid arteries (ICA) through cranial temporal bone windows; ophthalmic arteries (OA) and ICA siphon through orbital windows; terminal vertebral arteries (VA) and basilar arteries (BA) through foraminal window [4]. And carotid arteries were also insonated by using $4-\mathrm{MHz}$ probe, including extracranial ICA, external carotid arteries (ECA) [6]. All TCD examinations were performed by an experienced sonographer.

\section{Microembolic signals detection}

MES data were recorded and analyzed by an experienced observer, who was blinded to the clinical information. The following definitions for MES were used: typical visible and audible (click, chirp, whistle), short-duration, unidirectional, high-intensity signal $(\geq 5 \mathrm{~dB})$ within the Doppler flow spectrum with its occurrence at random in the cardiac cycle [7].

\section{Statistical analysis}

Student's t-test was performed to compare continuous variables; Pearson $\chi 2$ and Fisher's exact test were used to compare categorical variables between groups. A $p$ value less than 0.05 was considered statistically significant. All statistical analyses were done with SPSS 16.0 (SPSS Inc).

\section{Results}

During the study period, a total of 10,067 patients $(5162$ male, mean age $56.7 \pm 17.4$ ) received routine TCD examinations in our laboratory. Among them, 2305 (22.90 \%) patients had previous ischemic events or TIA.

MES was detected in 98 arteries of 78 patients, with a frequency of $0.77 \%$ of all the recruited patients. The MES positive arteries including MCA $(n=63)$, ACA $(n=8)$, SCA $(n=8)$, extracranial ICA $(n=8)$, PCA $(n=5)$,
$\mathrm{BA}(n=2)$, VA $(n=2), \mathrm{ECA}(n=1)$ and $\mathrm{OA}(n=1$, Table 1).

Sixty five (83.33\%) accidental emboli were presumed to come from arterial sources, including atherosclerotic cerebral or carotid artery stenosis $(n=45)$, moyamoya disease $(n=11$, Fig. 1$)$, intracranial arteries $(n=3)$ and Takayasu arteries $(n=3)$. Thirteen $(16.67 \%)$ emboli were presumed to come from cardiac sources, including atrial fibrillation $(n=3)$, artificial valves $(n=8)$, infective endocarditis $(n=2)$, patent foramen ovale $(n=2)$, and systemic Lupus Erythematosus $(n=1$, Table 2$)$.

In different diseases with accidental MES, the proportion of symptomatic patients and asymptomatic patients were different (Fisher exact test, $p<0.001$ ). In artificial valves disease, all patients with MES were asymptomatic, while in atherosclerotic cerebral or carotid artery stenosis, $66.67 \%(n=30)$ patients with MES were symptomatic (Table 2).

\section{Discussion}

In this study, accidental MES during routine TCD examinations were investigated in hospitalized patients with a large sample size. Overall, MES can be detected in 3 of 400 unselected hospitalized patients. A high percentage of MES (64.3\%) were detected in MCAs. It was not only because MCA is the common site of intracranial atherosclerosis and the largest artery that distributes blood to the cerebrum, but also the testing time of MCA was the longest, as the whole M1 segment could be detected by TCD.

The clinical significance of MES varied, as MES span a broad spectrum, ranging from harmless air bubbles to the large solid emboli [8]. In patients with artificial valves, none of the 8 patients with MES had the symptoms of cerebral ischemia, probably because the air bubbles produced by artificial valves are harmless. In atherosclerotic cerebral or carotid artery stenosis, however, $66.67 \%$

Table 1 Arteries with microembolic signals

\begin{tabular}{ll}
\hline Arteries MES(+) & $n(\%)$ \\
\hline MCA & $63(64.3)$ \\
SCA & $8(8.2)$ \\
ACA & $8(8.2)$ \\
ICAex & $8(8.2)$ \\
PCA & $5(5.1)$ \\
BA & $2(2.0)$ \\
VA & $2(2.0)$ \\
ECA & $1(1.0)$ \\
OA & $1(1.0)$ \\
\hline
\end{tabular}

MCA middle cerebral artery, SCA internal carotid artery siphon segment, $A C A$ anterior cerebral artery, ICAex extracranial internal carotid artery, $P C A$ posterior cerebral artery, $B A$ basilar artery, $V A$ vertebral artery,

$E C A$ external carotid artery, $O A$ ophthalmic artery 


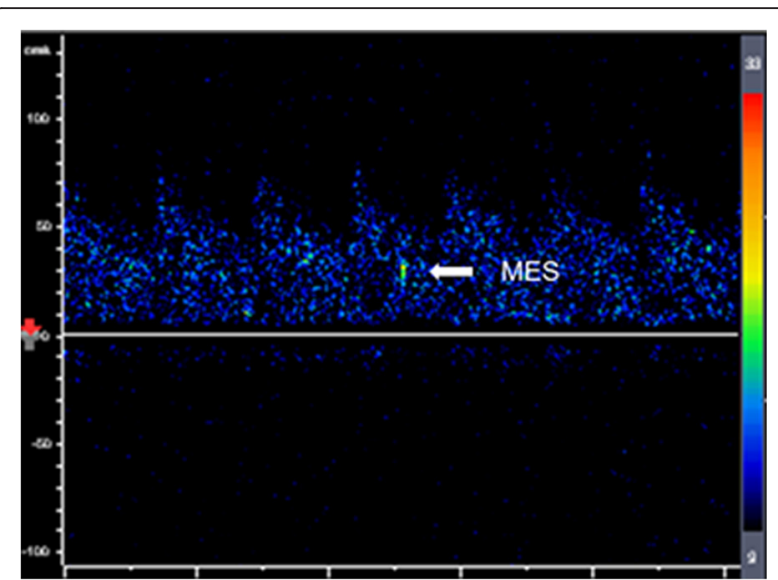

Fig. 1 Microembolic signal detected in a patient with moyamoya disease. Transcranial Doppler detected a microembolic signal in the right middle cerebral artery in a patient with moyamoya disease (arrow). MES, microembolic signal

patients with MES were symptomatic. It has been confirmed that the presence of MES was an independent predictor of future stroke in atherosclerotic occlusive cerebrovascular diseases [2, 7]. According to the practice standards for TCD, in patients with symptomatic and asymptomatic extracranial or intracranial large artery disease, the MES information could be used to detect, localize, and quantify cerebral embolization, which may be helpful to establish the diagnosis and change management strategy [5]. For example, one study demonstrated that in patients with MCA stenosis, the presence of an MES was associate with cerebral ischemia on diffusion weighted magnetic resonance imaging [9]. Thus the diffusion weighted imaging procedure might be helpful for these patients to detect cerebral ischemia. As to the therapy strategy, two randomized clinical trials demonstrated that in patients with symptomatic carotid stenosis $(\geq 50 \%)$ and

Table 2 Sources of accidental emboli

\begin{tabular}{lll}
\hline & Symptomatic & Asymptomatic \\
\hline Arterial Sources & 30 & 15 \\
$\begin{array}{l}\text { Atherosclerotic cerebral or carotid } \\
\text { artery stenosis }\end{array}$ & 9 & 2 \\
Moyamoya disease & 3 & 0 \\
Intracranial arteries & 0 & 3 \\
Takayasu arteries & & \\
Cardiac Sources & 2 & 1 \\
Atrial fibrillation & 0 & 8 \\
Artificial valves & 1 & 1 \\
Infective endocarditis & 1 & 0 \\
Patent foramen ovale & 1 & \\
Systemic Lupus Erythematosus & & \\
\hline
\end{tabular}

symptomatic cerebral or carotid artery stenosis, combination therapy with clopidogrel and aspirin was more effective than aspirin alone in reducing $\operatorname{MES}[10,11]$. Accidental MES in routine TCD examinations may also be an indicator for robust antiplatelet therapy.

It is interesting that MES were also observed in nonatherosclerotic artery stenosis including moyamoya disease, Takayasu's arteritis, and intracranial arteries. It provide evidence that artery-to-artery embolism may also play an important role in the underlying mechanism of ischemic stroke in these patients [12].

This study was a single-center retrospective investigation. There was a possibility of selection bias and information bias.

\section{Conclusions}

In conclusion, our study suggests that MES are not uncommon during routine TCD examinations, the clinical value of which varied in different diseases. MES detection during routine TCD examinations may be of potential clinical value in the management of patients.

\section{Abbreviations \\ ACA: anterior cerebral arteries; BA: basilar arteries; ECA: external carotid arteries; ICA: internal carotid arteries; MCA: middle cerebral arteries; MES: accidental microembolic signals; OA: ophthalmic arteries; PCA: posterior cerebral arteries; TCD: transcranial doppler; TIA: transient ischemic attack; VA: vertebral arteries.}

\section{Competing interests}

The authors declare no financial or other conflict of interests.

\section{Authors' contributions}

This study was designed by WHX and SG. JC, YQH conducted experiments and performed data analysis. The manuscript was written by JC and revision form WHX. All authors read and approved the final manuscript.

\section{Author details}

${ }^{1}$ Department of Neurology, Peking Union Medical College Hospital, Chinese Academy of Medical Sciences and Peking Union Medical College, Shuaifuyuan 1, Dongcheng District, Beijing 100730, China. ${ }^{2}$ Department of Neurology, Beijing Tiantan Hospital, Capital Medical University, Beijing 10050, China.

Received: 12 December 2015 Accepted: 3 February 2016

Published online: 12 April 2016

\section{References}

1. Spencer MP, Thomas Gl, Nicholls SC, Sauvage LR. Detection of middle cerebral artery emboli during carotid endarterectomy using transcranial Doppler ultrasonography. Stroke. 1990;21:415-23.

2. Ritter MA. Prevalence and prognostic impact of microembolic signals in arterial sources of embolism. J Neurol. 2008;255:953-61.

3. Purandare N, Voshaar RC, Morris J, Byrne JE, Wren J, Heller RF, et al. Asymptomatic spontaneous cerebral emboli predict cognitive and functional decline in dementia. Biol Psychiatry. 2007;62:339-44.

4. Ringelstein EB, Droste DW, Babikian VL, Evans DH, Grosset DG, Kaps M, et al. Consensus on microembolus detection by TCD. International Consensus Group on Microembolus Detection. Stroke. 1998;29:725-9.

5. Alexandrov AV, Sloan MA, Tegeler CH, Newell DN, Lumsden A, Garami Z, et al. Practice standards for transcranial Doppler (TCD) ultrasound. Part II. Clinical indications and expected outcomes. J Neuroimaging. 2012;22:215-24.

6. Sloan MA, Alexandrov AV, Tegeler CH, Spencer MP, Caplan LR, Feldmann E, et al. Assessment: transcranial Doppler ultrasonography: report of the 
Therapeutics and Technology Assessment Subcommittee of the American Academy of Neurology. Neurology. 2004;62:468-81.

7. Gao S, Wong KS, Hansberg T, Lam WW, Droste DW, Ringelstein EB. Microembolic signal predicts recurrent cerebral ischemic events in acute stroke patients with middle cerebral artery stenosis. Stroke. 2004;35:2832-6.

8. Choi Y, Saqqur M, Stewart E, Stephenson C, Roy J, Boulanger JM, et al. Relative energy index of microembolic signal can predict malignant microemboli. Stroke. 2010;41:700-6.

9. Wong KS, Gao S, Chan YL, Hansberg T, Lam WW, Droste DW, et al. Mechanisms of acute cerebral infarctions in patients with middle cerebral artery stenosis: a diffusion-weighted imaging and microemboli monitoring study. Ann Neurol. 2002;52:74-81.

10. Markus HS, Droste DW, Kaps M, Larrue V, Lees KR, Siebler M, et al. Dual antiplatelet therapy with clopidogrel and aspirin in symptomatic carotid stenosis evaluated using doppler embolic signal detection: the Clopidogrel and Aspirin for Reduction of Emboli in Symptomatic Carotid Stenosis (CARESS) trial. Circulation. 2005;111:2233-40.

11. Wong KS, Chen C, Fu J, Chang HM, Suwanwela NC, Huang YN, et al. Clopidogrel plus aspirin versus aspirin alone for reducing embolisation in patients with acute symptomatic cerebral or carotid artery stenosis (CLAIR study): a randomised, open-label, blinded-endpoint trial. Lancet Neurol. 2010:9:489-97.

12. Chen J, Duan L, Xu WH, Han YQ, Cui LY, Gao S. Microembolic signals predict cerebral ischaemic events in patients with moyamoya disease. Eur J Neurol. 2014;21:785-90.

\section{Submit your next manuscript to BioMed Central and we will help you at every step:}

- We accept pre-submission inquiries

- Our selector tool helps you to find the most relevant journal

- We provide round the clock customer support

- Convenient online submission

- Thorough peer review

- Inclusion in PubMed and all major indexing services

- Maximum visibility for your research

Submit your manuscript at www.biomedcentral.com/submit
Biomed Central 